\title{
MIELNIK'S PROBABILITY SPACES AND CHARACTERIZATION OF INNER PRODUCT SPACES
}

\author{
BY \\ C. V. STANOJEVIC
}

ABSTRACT. A characterization of inner product spaces is given in terms of Mielnik's probability function. The generalized parallelogram law is related to the functional equation $f+f \circ g=1$.

1. Introduction. In the classical approach of G. Birkhoff and J. von Neumann [1] the main problem was to find a set of axioms for the quantum logic that would guarantee the existence of a representation of quantum logic propositions by orthogonal projectors in a Hilbert space. C. Piron [2] assumed weak semimodularity and showed that yes-no measurements can be represented by projectors in some unitary spaces.

In [3] B. Mielnik pointed out the insufficiency of the classical approach and proposed a geometric approach to the foundation of general quantum mechanics. For that purpose he introduced an abstract space of states as a setting for his geometric theory of quantum states.

Let $S$ be a nonempty set, and let $p$ be a real-valued function defined on $S \times$ $S$ such that

$$
\begin{gathered}
0 \leq p(a, b) \leq 1 \quad \text { and } \quad a=b \Leftrightarrow p(a, b)=1 \\
p(a, b)=p(b, a) \text { for all } a, b \in S .
\end{gathered}
$$

Two elements $a$ and $b$ in $S$ are orthogonal if $p(a, b)=0$. A subset $R$ of $S$ is an orthogonal system if any two distinct elements of $R$ are orthogonal. From Zorn's lemma it follows that there exists a maximal orthogonal system $B$ which is called a basis in $S$.

Let $B$ be a basis and $F_{B}$ be the class of all finite subsets $F$ of $B$. Define $p(a, F)=\Sigma_{b \in F} p(a, b)$ for all $a \in S$. Then the following property of $(S, p)$ is also postulated:

(C) For each basis $B$ and for each $a \in S$,

Received by the editors March 10, 1972.

AMS (MOS) subject classifications (1970). Primary 46C10, 81A 10.

Key words and phrases. Inner product spaces, foundations of general quantum mechanics. 


$$
\sup _{F \in F_{B}} p(a, F)=1
$$

Any pair $(S, p)$ satisfying axioms $(A),(B)$ and $(C)$ is called a probability space.

Let $B_{1}$ and $B_{2}$ be two bases in $S$; then, as shown in [3], $B_{1}$ and $B_{2}$ have the same cardinal number. The cardinal number is called the dimension of $(S, p)$.

The existence of a representation of states by vectors in a Hilbert space imposes strong limitations on the geometric structure of the space of states. This space must be a Hilbert space over one of the three fields: real numbers, complex numbers or quaternions. An analogous stiuation arises in connection with Mielnik's theory. A particular probability space structure, imposed on a subset $S$ of a normed linear space $N$, can turn $N$ into an inner product space. For instance, in [4] we have the result:

Let $S$ be the unit sphere of a normed real linear space $N$ and let $p(a, b)=$ $1 / 4\|a+b\|^{2}$ for $a, b$ in $S$. Then $N$ is an inner product space if and only if $(S, p)$ is a probability space of dimension 2 .

The theorems that follow indicate that the above result is not a curious observation only.

Theorem 1.1. Let $S$ be the unit sphere of a normed real linear space $N$. If $(S, p)$ is a probability space of dimension 2 sucb that

$$
p(x, y) \leq 1 / 4\|x+y\|^{2}, \text { for all } x, y \in S,
$$

then $N$ is an inner product space.

Proof. Put $y=-x$ in (1.1). Then $p(x,-x) \leq 0$. But from axiom (A) we get $p(x,-x)=0$, for all $x \in S$. Therefore, each basis in $S$ is of the form $\{y,-y\}$ for $(S, p)$ is of dimension 2. Hence, according to the axiom (C), $p(x, y)+p(x,-y)=1$. Thus, from (1.1), we have $1 / 4\|x+y\|^{2}+1 / 4\|x-y\|^{2} \geq 1$ for all $x, y$ in $S$.

Recalling I. J. Schoenberg's result [5], we see that $N$ is an inner product space.

The converse of Theorem (1.1) is plain.

In the next theorem we can use a slightly weakened axiom (A):

$$
0 \leq p^{\prime}(a, b) \leq 1 \text { and } p^{\prime}(a, b)=1 \Rightarrow a=b .
$$

Theorem 1.2. Let $S$ be the unit sphere of a normed real linear space N. If $(S, p)$ is a probability space such that

$$
p^{\prime}(x, y) \geq 1 / 4\|x+y\|^{2}
$$

for all $x, y$ in $S$, and the dimension of $\left(S, p^{\prime}\right)$ is 2 , then $N$ is an inner product space. 
Proof. The condition (1.2) and $p^{\prime}(a, b)=1 \Rightarrow a=b$ imply axiom (A). Let $y=$ $x$, then from $(1.2)$ it follows that $p(x, x) \geq 1$. But $p(x, x) \leq 1$, so $p(x, x)=1$. In other words, (1.2) implies that $\left(S, p^{\prime}\right)$ is $(S, p)$.

Since $\left(S, p^{\prime}\right)$ is of dimension 2 , we have $p^{\prime}(x, y)+p^{\prime}(x,-y)=1$, or according to $(1.2), 1 / 4\|x+y\|^{2}+1 / 4\|x-y\|^{2} \leq 1$.

Using again I. J. Schoenberg's condition we get that $N$ is an inner product space.

The above results motivate an effort to characterize inner product spaces in terms of probability spaces in the sense of B. Mielnik.

In this paper we shall give a necessary and sufficient condition for $N$ to be an inner product space in terms of probability space structure imposed over the unit sphere $S$ of $N$.

2. Lemmas. Let $[0,2]$ be the domain and $[0,1]$ the range of $f$, and let $f$ be continuous and increasing, with $f(0)=0$ and $f(2)=1$. The class of all such functions we denote by $F$.

Let $[0,2]$ be both the domain and the range of continuous and decreasing function $g$, and let $g(0)=2, g(2)=0$. The class of these functions we denote by G.

For our purpose we consider those $f \in F$ and $g \in G$ that satisfy the functional equation

$$
f+f \circ g=1
$$

where $(f \circ g)(t)=f(g(t))$.

Lemma 2.1. Let $f \in F$. Then there exists a $g \in G$ such that (2.1) bolds.

Proof. Direct verification shows that

$$
g(t)=f^{-1}(1-f(t)), \quad t \in[0,2],
$$

satisfies (2.1) and $g \in G$.

Lemma 2.2. Let $g \in G$. Then (2.1) bas a solution $f \in F$ if and only if $g$ is an involution, i.e. $g=g^{-1}$.

Proof. Suppose that $f$ satisfies (2.1), and change $t$ by $g(t)$,

$$
f(g(t))+f(g(g(t)))=1 .
$$

Hence $f(t)=f(g(g(t)))=f\left(g_{2}(t)\right)$, where $g_{2}=g \circ g$. Since $g_{2}(t)$ increases from $0=$ $g_{2}(0)$ to $2=g_{2}(2)$, and $f$ is univalent, we obtain $t=g_{2}(t)$, or $g^{-1}(t)=g(t), t \in$ $[0,2]$. Thus $g=g^{-1}$ is necessary.

Assume that $g=g^{-1}$. We shall prove that all solutions of $(2.1)$ can be obtained in the following way: Let $z$ be such that 


$$
g(z)=z
$$

Choose $f(t)$ in $[0, z]$ such that $f$ is increasing in $[0, z]$ and

$$
f(0)=0, \quad f(z)=1 / 2 .
$$

Define $f(t)$ in $[z, 1]$ by

$$
f(t)=1-f^{-1}\left(g^{-1}(t)\right), \quad t \in[z, 1]
$$

Since $g^{-1}(t)$ decreases from $z$ to 0 , as $t$ increases from $z$ to 2 , it follows that $f$ defined by (2.2) increases from $1 / 2$ to 1 , and therefore $f \in F$. If $t \in[0, z]$, then $g(t) \in[z, 2]$. Changing $t$ by $g(t)$ in (2.2) we get $f(g(t))=1-f(t)$. That completes the proof of Lemma 2.2.

Example 2.1. Let $b \in G$. If

$$
g(t)=b^{-1}\left(2-\lg \left[e^{2}-e^{2-b(x)}+1\right]\right)
$$

then

$$
f(t)=\left(e^{2-b(t)}-1\right) /\left(e^{2}-1\right)
$$

is the solution of (2.1).

Example 2.2. For

$$
h(t)=-\lg \left[1 / 4 t^{2}\left(1-e^{-2}\right)+e^{-2}\right]
$$

we have

$$
f(t)=1 / 4 t^{2}
$$

Lemma 2.3 (D. A. Senechalle). Let $N$ be a normed real linear space and let $S=\{x \mid\|x\|=1\}$. Then $N$ is an inner product space if and only if there exists some real function $F$ in $[0,2]$ such that

$$
F(\|x+y\|)=\|x-y\|
$$

for all $x, y \in S$.

Proof. See [6].

In order to use Mielnik's geometry of quantum states for characterization of inner product spaces we need the generalized parallellogram law in an explicit form. With the help of $(2.1)$ we can give such a characterization of inner product spaces.

Lemma 2.4. Let $N$ be normed real linear space, and $S=\{x \mid\|x\|=1\}$. Then $N$ is an inner product space if and only if 


$$
f(\|x+y\|)+f(\|x-y\|)=1
$$

for some $f \in F$ and all $x, y \in S$.

Proof. Assume (2.4), then

$$
\|x-y\|=f^{-1}(1-f(\|x+y\|))
$$

According to Lemma 2.1 there is a $g \in G$ such that $g(\|x+y\|)=f^{-1}(1-f(\|x+y\|))$, or $\|x-y\|=g(\|x+y\|)$. Applying, now, the Senechalle result from Lemma 2.3 we have that (2.4) implies the existence of an inner product. Suppose $N$ is an inner product space. It suffices to show that for some $f \in F$ and $g \in G,(2.1)$ i.e. (2.4) becomes

$$
\|x+y\|^{2} / 4+\|x-y\|^{2} / 4=1 .
$$

Indeed, let $f(t)=(t / 2)^{2}, t \in[0,2]$, then there is $g(t)=2 \sqrt{1-(t / 2)^{2}}$ such that (2.1), i.e. (2.4), reduces to (2.5) which is a well-known condition of M. M. Day's [7].

Example 2.3. Using Example 2.1 we have that a necessary and sufficient condition for $N$ to be an inner product space is that

$$
e^{-b(\|x+y\|)}+e^{-b(\|x-y\|)}=1+e^{-2}
$$

for some $b \in G$, and all $x, y \in S$.

Example 2.4. For $b$ as in Example 2.2, the condition (2.6) reduces to that of M. M. Day.

3. Probability spaces and inner product spaces. It remains to determine the extent of limitation that a very general probability space structure imposes upon a normed real linear space $N$. We shall show that a certain probability space structure imposed upon $S=\{x \mid\|x\|=1\} \subset N$, makes $N$ into an inner product space.

Theorem 3.1. Let $N$ be a normed real linear space, $S=\{x \mid\|x\|=1\}$ and let $p(x, y)=f(\|x+y\|)$, where $f \in F$.

Then $N$ is an inner product space if and only if for some $f \in F,(S, p)$ is a probability space of dimension 2.

Proof. Suppose that $(S, p)$ is a probability space of dimension 2. From the axiom (C) we have that $f(\|x+y\|)+f(i \mid x-y \|)=1$ holds for all $x, y \in S$. Then according to Lemma $2.4, N$ is an inner product space.

Assume that $N$ is an inner product space. Then axiom $(\mathrm{A})$ holds. First $0 \leq$ $f(\|x+y\|) \leq 1$, for $f \in F$. Further, if $x=y$, then $p(x, y)=f(2)=1$; if $f(\|x+y\|)=$ 1 , then $\|x+y\|=f^{-1}(1)=2$; but in any inner product space we have that $\|x\|=$ $\|y\|=1$ and $\|x+y\|^{2}=4$ imply that $x=y$. 
The axiom (B) is clearly satisfied. From $f(\|x+y\|)=0$, we get $\|x+y\|=0$, i.e. that $x$ and $-x$ are orthogonal. Hence the bases are of the form $\{x,-x\}$. Applying Lemma 2.4 , i.e. that for some $f \in F$,

$$
f(\|x+y\|)+f(\|x-y\|)=1
$$

holds for all $x, y \in S$, we see that the axiom (C) is satisfied. This completes the proof.

Example 3.1. Let $b$ satisfy the conditions of Example 2.1 and let $N$ and $S$ be as in Theorem 3.1.

Then $N$ is an inner product space if and only if

$$
\left(S,\left(e^{2-b(\|x+y\|)}-1\right) /\left(e^{2}-1\right)\right)
$$

is a probability space of dimension 2 , for some $b \in G$.

Example 3.2. Let the conditions of Example 3.1 be satisfied. Then for

$$
b(t)=-\lg \left[1 / 4 t^{2}\left(1-e^{-2}\right)+e^{-2}\right]
$$

we have the result proved in [4].

4. Remarks.

4.1. In view of Theorem 3.1 the condition (1.1) becomes

$$
p(x, y) \leq f(\|x+y\|)
$$

and (1.2) becomes

$$
p^{\prime}(x, y) \geq f(\|x+y\|) .
$$

4.2. Lemma 2.4 is essentially a Lorch type of condition [8], and it is related to M. M. Day's condition in the same way as the original Lorch condition is to that of P. Jordan and J. von Neumann [9].

The condition (2:4) of Lemma 2.4 is a direct generalization of the well-known parallelogram law [9], and for our purpose it is more natural than that of Senechalle. One can get the cond ition (2.4) from Senechalle's condition (2.3) using Lemma 2.2.

4.3. If in Theorem 3.1 we omit the condition that $f$ is increasing, we can still get some restrictions about $N$.

Let $f$ be continuous in $[0,2], f(2)=1$, and let 0 be the only point such that $f(0)=0$. We have the following result.

Theorem 4.1. Let $N$ be a normed real linear space, $S=\{x \mid\|x\|=1\}$ and let $f$ be as above. If $(S, f(\|x+y\|))$ is a probability space of dimension 2 , then $N$ is uniformly convex.

Proof. Since $(S, f(\|x+y\|))$ is a probability space of dimension 2, we have 


$$
f(\|x+y\|)+f(\|x-y\|)=1
$$

Let $\left\|x_{n}\right\|=1$, and $\left\|y_{n}\right\|=1, n=1,2, \ldots$; furthermore, assume that $\left\|\left(x_{n}+y_{n}\right) / 2\right\| \rightarrow$ 1 as $n \rightarrow \infty$. Then from (4.1) we have

$$
f\left(\left\|x_{n}+y_{n}\right\|\right)+f\left(\left\|x_{n}-y_{n}\right\|\right)=1
$$

and

$$
\lim _{n \rightarrow \infty} f\left(\left\|x_{n}+y_{n}\right\|\right)+\lim _{n \rightarrow \infty} f\left(\left\|x_{n}-y_{n}\right\|\right)=1
$$

or

$$
\lim _{n \rightarrow \infty} f\left(\left\|x_{n}-y_{n}\right\|\right)=1-f(2)
$$

for $f$ is continuous at 2. Finally, $\lim _{n \rightarrow \infty}\left\|x_{n}-y_{n}\right\|=0$, for $f$ is continuous at 0 , $f(2)=1$ and 0 is the only point such that $f(0)=0$. This proves that $N$ is uniformly convex.

4.4. It appears that if we impose limitations of the Piron kind or the Mielnik kind, the space in which representation is taking place undergoes some restrictions, which range from uniformly convex (Theorem 4.1 ) to the existence of an inner product (e.g., Theorem 3.1).

It should be pointed out that this remark concerns only Mielnik's probability spaces of dimension 2. In the case of Mielnik's probability space of dimension $\geq 3$ with $p(x, y)=f(\|x+y\|), N$ will not necessarily be an inner product space.

4.5. Consider a linear topological space $T$ and a suitably chosen subset $S$ of $T$. One could try to find the conditions for $p(x, y)$ such that if $(S, p)$ is a probability space then $T$ is a normed linear space. For some indications in this direction see the second Mielnik paper [10].

4.6. Inequalities (4.1) and (4.2) indicate that $p(x, y)$ might be related to the semi-inner product in the sense of G. Lumer[11] and J. R. Giles [12]. It would be of interest to know what the implications might be of the relations between the geometry of quantum states and characterizations of semi-inner product spaces.

The author takes pleasure to express his gratitude to the referee for his very valuable remarks. .

\section{REFERENCES}

1. G. Birkhoff and J. von Neumann, The logic of quantum mechanics, Ann of Math. (2) 37 (1936), 823-843.

2. C. Piron, Axiomatique quantique, Helv. Phys. Acta 37 (1964), 439-468. MR 34 \#3894.

3. B. Mielnik, Geometry of quantum states, Comm. Math. Phys. 9 (1968), 55-80. MR 37 \#7 156.

4. C. V. Stanojevic, Mielnik's probability manifolds and inner product spaces, Bull. 
Acad. Polon. Sci.»Sér. Sci. Math. Astronom. Phys. 18 (1970), 531-532. MR 42 \#8259.

5. I. J. Schoenberg, A remark on M. M. Day's characterization of inner product spaces and a conjecture of L. M. Blumenthal, Proc. Amer. Math. Soc. 3 (1952), 961-964. MR 14, 564.

6. D. A. Senechalle, A characterization of inner product spaces, Proc. Amer. Math. Soc. 19 (1968), 1306-1312. MR 39 \#761.

7. M. M. Day, Some characterizations of inner product spaces, Trans. Amer. Math. Soc. 62 (1947), 320-337. MR 9, 192.

8. E. R. Lorch, On certain implications which characterize Hilbert space, Ann. of Math. (2) 49 (1948), 523-532. MR 10, 129.

9. P. Jordan and J. von Neumann, On inner products in linear spaces, Ann. of Math. (2) 36 (1935), 719-732.

10. B. Mielnik, Theory of filters, Comm. Math. Phys. 15 (1969), 1-46. MR $41 \# 3004$.

11. G. Lumer, Semi-inner product spaces, Trans. Amer. Math. Soc. 100 (1961), 29-43. MR 24 \#A2860.

12. J. R. Giles, Classes of semi-inner product spaces, Trans. Amer. Math. Soc. 129 (1967), 436-446. MR $36 \# 663$.

DEPARTMENT OF MATHEMATICS, UNIVERSITY OF MISSOURI, ROLLA, MISSOURI 65401 